\title{
Editorial
}

\section{Adaptive and Sequential Methods for Clinical Trials}

\author{
Yichuan Zhao, ${ }^{1}$ Zhengjia Chen, ${ }^{2}$ Xuelin Huang, ${ }^{3}$ and Mourad Tighiouart ${ }^{4}$ \\ ${ }^{1}$ Department of Mathematics \& Statistics, Georgia State University, Atlanta, GA 30303, USA \\ ${ }^{2}$ Department of Biostatistics and Bioinformatics, Emory University, Atlanta, GA 30322, USA \\ ${ }^{3}$ Department of Biostatistics, The University of Texas MD Anderson Cancer Center, Houston, \\ TX 77030, USA \\ ${ }^{4}$ Samuel Oschin Comprehensive Cancer Institute, Cedars-Sinai Medical Center, Los Angeles, \\ CA 90048, USA \\ Correspondence should be addressed to Yichuan Zhao; yichuan@gsu.edu
}

Received 31 October 2012; Accepted 31 October 2012

Copyright (C) 2013 Yichuan Zhao et al. This is an open access article distributed under the Creative Commons Attribution License, which permits unrestricted use, distribution, and reproduction in any medium, provided the original work is properly cited.

The clinical trial, a prospective evaluation of the effect of interventions in humans under prespecified conditions, is a standard and integral part of modern medicine. Many adaptive and sequential approaches have been proposed for clinical trials, which allow for modifications to an ongoing trial without undermining the validity and integrity of the trial. The application of adaptive and sequential methods in clinical trials has significantly improved the flexibility, efficiency, therapeutic effect, and validity of such trials.

This special issue describes state-of-the-art statistical research in adaptive and sequential methods and the application of such methods in clinical trials. It provides 1 review article and 5 research articles contributed by some of the leading experts in this field. The review article gives a comprehensive overview of the outstanding methodology in the current literature that is related to adaptive and sequential clinical trials, while each of the 5 research articles addresses specific critical issues in contemporary clinical trials, as summarized below.

In the review paper "Methodology and Application of Adaptive and Sequential Approaches in Contemporary Clinical Trials," by Z. Chen et al., the most distinguished and applicable adaptive and sequential approaches, especially novel designs, are reviewed, compared, and contrasted according to the phase of clinical trial (phases I, II, and III) to which they are applied. The future directions of the related areas of research are also explored and discussed.

The research article entitled "Exact group sequential methods for estimating a binomial proportion," by Z. Chen and $\mathrm{X}$. Chen, first reviews existing sequential methods for estimating a binomial proportion. A new family of group sequential sampling schemes is then proposed for estimating a binomial proportion with a prescribed margin of error, which achieves unprecedented efficiency while guaranteeing prespecified confidence levels.

The research article entitled "Two-stage adaptive optimal design with fixed first-stage sample size," by A. Lane and N. Flournoy, proposes a two-stage adaptive optimal design with a fixed first-stage sample size, as applied to a small pilot study of fixed size that is to be followed by a much larger experiment. The authors study the large sample behavior of their design by assuming a nonlinear regression model with normal errors and explicitly deriving the asymptotic distribution of the maximum likelihood estimate.

In the research paper "Escalation with overdose control using ordinal toxicity grades for cancer phase I clinical trials," by M. Tighiouart et al., the authors extend a Bayesian adaptive phase I clinical trial by introducing an intermediate-grade toxicity and show that the efficiency and safety of the trial are maintained and fewer patients are overdosed.

The research paper "Incorporating a patient dichotomous characteristic in cancer phase I clinical trials using escalation with overdose control," by M. Tighiouart et al., describes a design for phase I clinical trials in cancer that takes into account heterogeneity among patients, which is thought to be related to treatment susceptibility, and reduces the number of patients being overdosed. 
In the research paper "Number of patients per cohort and sample size considerations using dose escalation with overdose control," M. Tighiouart and A. Rogatko compare the safety and efficiency of trials designed with three or only one patient per cohort and present the number of patients needed to design a trial to achieve a given accuracy of the estimate of the maximum tolerated dose.

As the editors of this special issue, we hope that readers of this special journal issue will find these articles representative of the contributions of this important research field to clinical trials, in terms of research methodology and its many practical applications. We thank the authors and reviewers for their significant contributions to the articles in this special issue. We also extend our thanks to the Hindis Publishing Corporation for their professional and efficient service.

Yichuan Zhao

Zhengjia Chen

Xuelin Huang

Mourad Tighiouart 


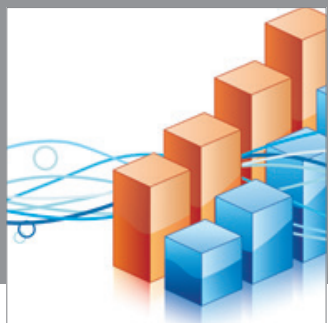

Advances in

Operations Research

mansans

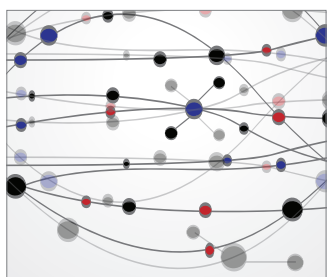

The Scientific World Journal
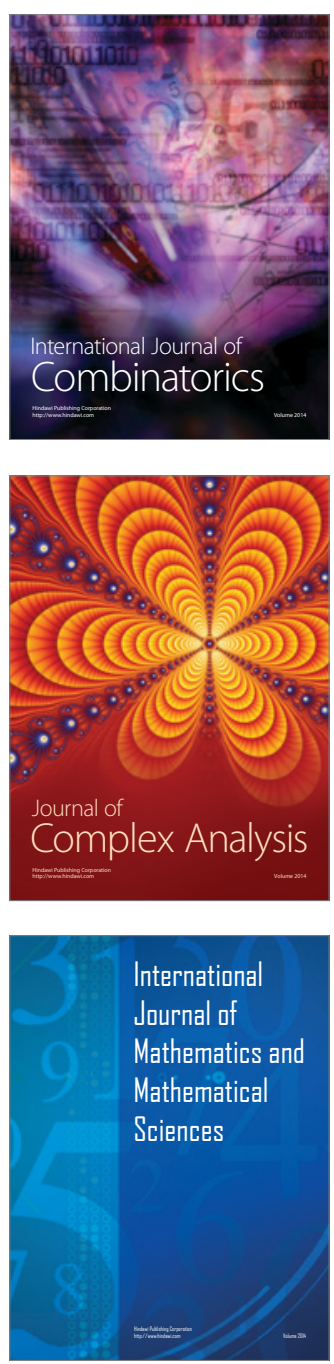
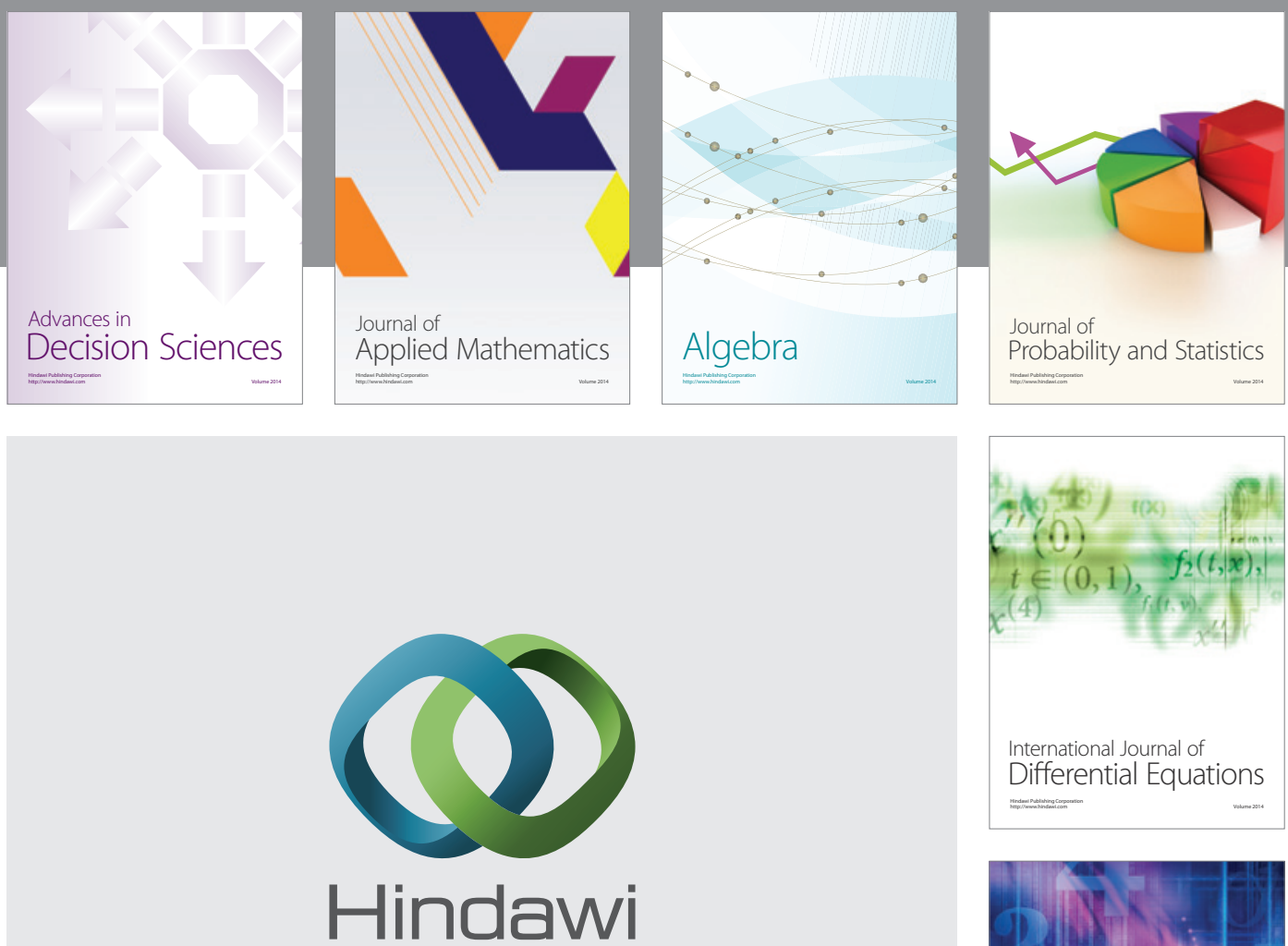

Submit your manuscripts at http://www.hindawi.com
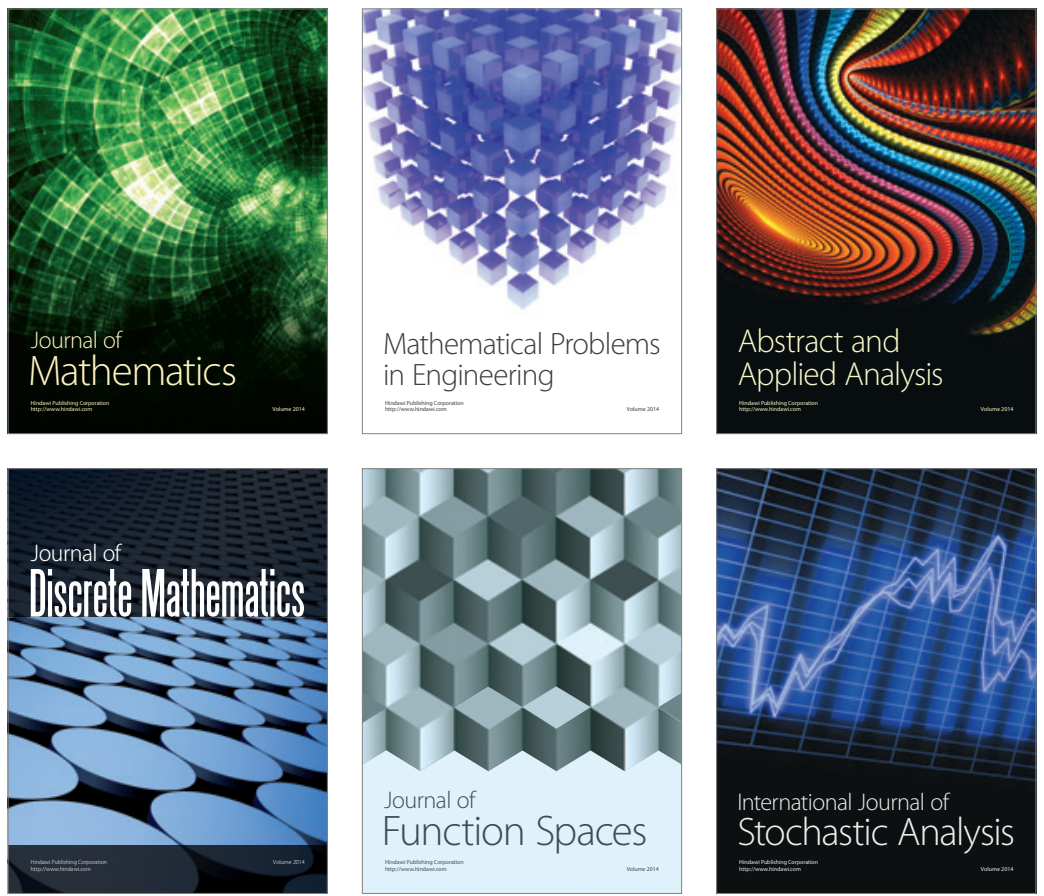

Journal of

Function Spaces

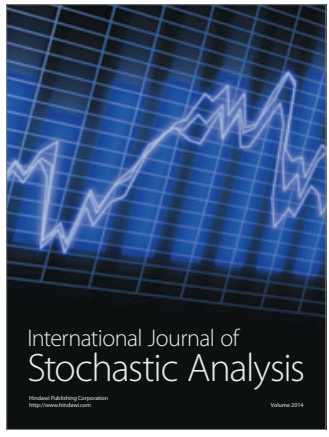

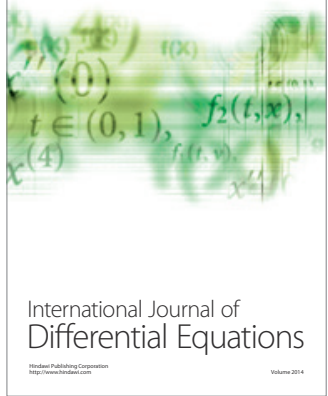
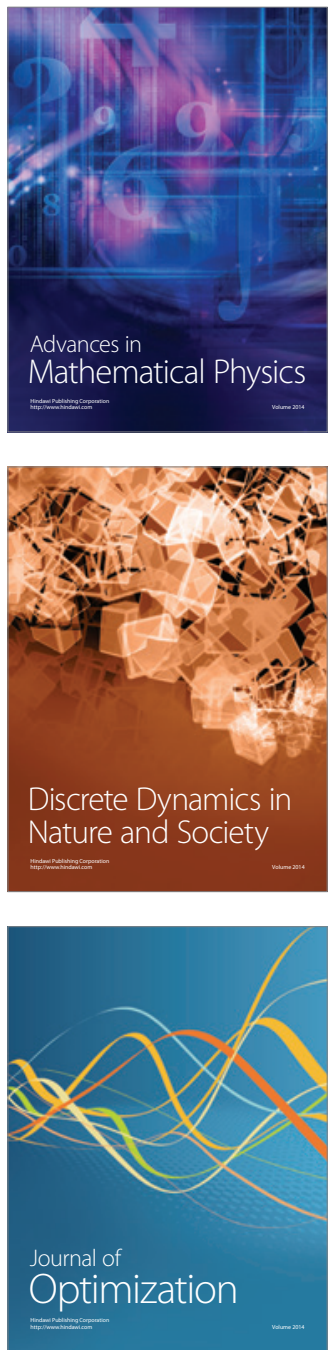